\title{
Big data applications for healthcare: preface to special issue
}

\author{
Sabah Mohammed ${ }^{1}$. Tai Hoon Kim ${ }^{1}$
}

Published online: 11 October 2016

(C) Springer Science+Business Media New York 2016

One of the vital challenges to big data in healthcare is the development of proper analytics tools applicable to unstructured clinical data (e.g., stream data collected from wearable vital signs sensors, health-related blogs, online medical publications, pharmacovigilance, and textual discharge summaries for referral purposes) which can reach massive proportions over time. These tools need to be architected for clinicians to enable them to see far past what the traditional data analytics solutions provide. Most of the current data analytics tools and techniques used in healthcare are borrowed from eCommerce with a plethora of metrics for uncertain complex workflows that often confuse clinicians making their use almost impossible. There are many other challenges for the use big data in healthcare to enable its analytics to predict epidemics, cure disease, improve quality-of-life, and avoid preventable deaths. The most important challenge is the lack of pre-defined structure. The difficulty with big data is that it is not trivial to find needed data within that massive, unstructured data store. It is not like the legacy relational database which essentially comes with a clear structure and a method to access the data [1]. With big data, there are no traditional schemas and, therefore, not much guidance. With a relational database, a simple, structured query language (i.e., SQL) pulls the needed data using a sophisticated query engine optimized for finding data [2]. The potential of big data allows us to hope to slow the ever-increasing costs of care, help providers practice more effective medicine, empower patients and caregivers, support fitness and preventive self-care, and to develop more personalized medicine. This potential can be achieve using the

\footnotetext{
Sabah Mohammed

sabah.mohammed@lakeheadu.ca

Tai Hoon Kim

taihoonn@daum.net

1 Thunder Bay, Canada
} 
right information technology tools and methods as well as solving the most important challenges, including privacy protection [3].

The following 16 papers have been selected for inclusion in this special issue:

1. Health Fog: a novel framework for health and wellness applications, doi:10.1007/ s11227-016-1634-X

2. Optimization strategy of Hadoop small file storage for big data in healthcare, doi:10.1007/s11227-015-1462-4

3. Improving the classification performance of biological imbalanced datasets by swarm optimization algorithms, doi:10.1007/s11227-015-1541-6

4. Leveraging information security and computational trust for cybersecurity, doi:10. 1007/s11227-015-1543-4

5. Recent advances in metaheuristic algorithms: Does the Makara dragon exist?, doi:10.1007/s11227-015-1592-8

6. Disclosing user relationships in email networks, doi:10.1007/s11227-015-15247

7. NSPRING: the SPRING extension for subsequence matching of time series supporting normalization, doi:10.1007/s11227-015-1525-6

8. A privacy preserving three-factor authentication protocol for e-Health clouds, doi:10.1007/s11227-015-1610-X

9. Discovering sub-patterns from time series using a normalized cross-match algorithm, doi:10.1007/s11227-016-1632-z

10. GPU-enabled back-propagation artificial neural network for digit recognition in parallel, doi:10.1007/s11227-016-1633-y

11. A time series pre-processing methodology with statistical and spectral analysis for classifying non-stationary stochastic biosignals, doi:10.1007/s11227-016-16359

12. An efficient Session_Weight load balancing and scheduling methodology for high-quality telehealth care service based on WebRTC, doi:10.1007/s11227-0161636- 8

13. Improvised methods for tackling big data stream mining challenges: case study of human activity recognition, doi:10.1007/s11227-016-1639-5

14. Finding approximate solutions of NP-hard optimization and TSP problems using elephant search algorithm, doi:10.1007/s11227-016-1739-2

15. Towards implementation of residual-feedback GMDH neural network on parallel GPU memory guided by a regression curve, doi:10.1007/s11227-016-1740-9

16. Autonomous path based data acquisition in sensor networks, doi:10.1007/s11227016-1745-4.

\section{References}

1. Koumpourosm Y (2014) Big data in healthcare. In: Healthcare Administration: Concepts, Methodologies, Tools, and Applications, 1st edn. IGI Global (ISBN-13: 978-1466663398)

2. Adamson D (2015) Big data in healthcare made simple: where it stands today and where it's going. Online Article https://www.healthcatalyst.com/big-data-in-healthcare-made-simple

3. Fiaidhi J, Kuziemsky C, Mohammed S, Weber J, Topaloglou T (2016) Emerging IT trends in healthcare and well-being. IT Prof 18(3):9-13 